\title{
A study of product quality in the electricity network in the province of Burgos
}

\author{
J. Sagredo González, V. Abad San Martín and J. González de la Viuda \\ Departamento de Ingeniería Electromecánica \\ Escuela Politécnica Superior, Universidad de Burgos \\ Avda. Cantabria S/N, 09006 Burgos (España) \\ phone:+34 947259347, fax:+34 947 259088, e-mail: jsgpol@ubu.es, vaspol@ubu.es
}

\begin{abstract}
Changes in the regulation of electrical systems have meant a reorganisation of the firms in the sector, subdividing them into different areas of action. Distribution firms, in addition to supplying energy, are now responsible for monitoring the "service quality" with which the product reaches the client and for communicating to the Ministry of Economics (Minister for Energy Policy and Mines) and to the corresponding Administrations information as to service quality established in the decree-law.
\end{abstract}

The first of its contents refers to continuity of the service, for which measurement and control procedures have already been passed, such that we now have the first published data at community level. As regards product quality, the aforementioned Royal Decree indicates that the characteristics of the wave must be those specified in norm UNE-EN 50160, while the corresponding measurement and control procedures, for which there are already some draft versions, are still pending approval.

The paper focuses on the quality of the product and aims to harmonise the steps established in some of the draft versions to delimit margins, establish measurement periods and suggest a format for the presentation and interchange of data.

In collaboration with the electricity company IBERDROLA, we have begun to acquire measurements, to carry out a "precompliance" study in the province of Burgos.

\section{Keywords}

Power Quality, voltage wave, distribution, standard.

\section{Introduction}

Once the universal supply of electricity has been guaranteed, increasing interest is being paid to the properties of this supply, demanding greater quality of the supply companies, just as occurs with any commercial product.
R.D.1955/2000 is a novel Royal Decree, where for the first time in regulation of electricity a relationship is established between what consumers pay for their electricity supply and the quality with which they receive it. Given that $80 \%$ of problems occur in the distribution network, it is on this sector on which we focus our paper.

The quality of service includes various aspects detailed in the Royal Decree, as the following table shows:

TABLE I. Royal Decree 1955/2000 Quality Service.

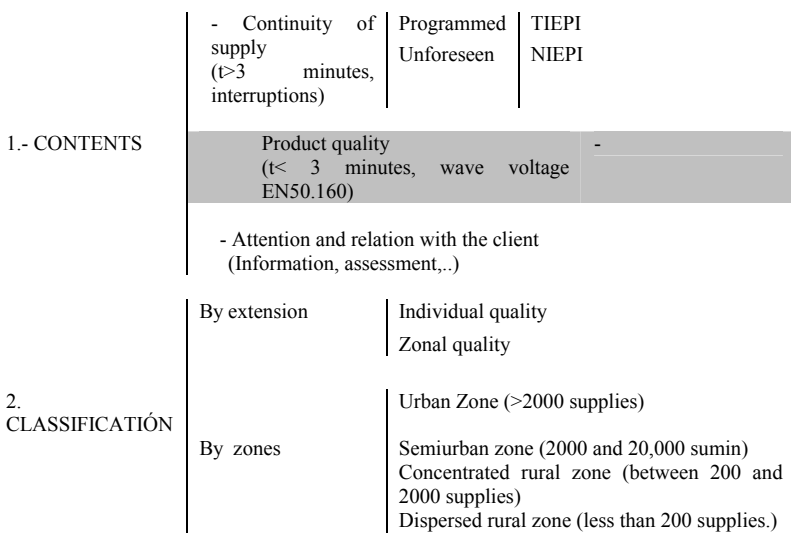

Distribution companies must prepare detailed annual information, and therefore a homogenous procedure is required for measurement and control for all companies, which, in turn, will make it possible for an external company to audit the data.

There is currently no total quality index similar to the North American "Power Premium Grades", but rather the different contents which encompass service quality are measured independently. 


\section{The current state of norms and legislation}

At the present moment, if one looks at the norms regarding Power Quality, one sees that many of the regulations are drafts and they do not establish clear criteria as to limits or any other aspects. In the USA, IEEE standard 1159 is responsible for developing its 3 sections: the first includes the specifications of measurement equipment, the second the definition of the events and their characteristics, while the third develops a file format for wave quality data known as Power Quality Data Interchange Format (PQDIF)[1].

In Spain, where electric wave quality is concerned, there are two essential documents: the R.D. 1955/2000[2] and the norm UNE-EN-50160[3]. In addition, at a European level, norm IEC 61000 establishes all those aspects relative to conducted disturbances, while other organisms such as UNESA[4], UNIPEDE or EURELECTRIC have prepared their own documents.

\section{A. The Royal Decree 1955/2000.}

This Royal Decree, for the first time, obliged distribution companies (DC) to measure and inform as to the quality of their service.

This document sets the parameters and calls on the DCs, through their association UNESA, to present procedures for measurement and control. Complying with the established deadline, UNESA made a proposal to the National Energy Commission (CNE), assigned by the Ministry for the Economy to approve it.

The CNE gave a favourable report on the proposal relative to the continuity of supply and the procedures were published in the BOE of 13 April, 2002 and in March, 2002, the first reports were made public.

As regards the question we are dealing with, product quality, the report of the CNE was unfavourable with respect to the proposal for product quality, pending the passing of IEC norm IEC 61000-4-30[5], where the requirements of the necessary measurement equipment are specified. This norm has just been published, in February, 2003.

In the section on customer service, the only point of note is that the deadlines to be complied with by the DCs in the different classes of supply are indicated, but no specific procedure is needed.

\section{B. Norm EN 50160}

This norm describes the characteristics of the wave voltage in the general distribution networks. It specifies thirteen parameters, of which, for product quality we consider those shown in table I.
TABLE II. EN-50160 abstract for LV.

\begin{tabular}{|c|c|c|c|c|}
\hline Param & $\begin{array}{l}\text { Nom. } \\
\text { value }\end{array}$ & $\begin{array}{c}\text { Limit } \\
\text { Interval }\end{array}$ & $\begin{array}{c}\text { \% of } \\
\text { Measures }\end{array}$ & $\begin{array}{c}\text { Promed. } \\
\text { Time }\end{array}$ \\
\hline Frequency & $50 \mathrm{~Hz}$ & $\begin{array}{c} \pm 1 \% \\
+4 /-6 \%\end{array}$ & $\begin{array}{c}95 \% \\
100 \%\end{array}$ & $10 \mathrm{~s}$ \\
\hline $\begin{array}{l}\text { Voltage } \\
\text { magnitude }\end{array}$ & $230 \mathrm{~V}$ & $\begin{array}{l} \pm 10 \% \\
\pm 7 \%\end{array}$ & $95 \%$ & $10 \mathrm{~min}$ \\
\hline Flicker Plt & & 1 & $95 \%$ & 2 hours \\
\hline $\mathrm{THD}_{\mathrm{U}}$ & & $8 \%$ & $95 \%$ & $10 \mathrm{~min}$ \\
\hline $\begin{array}{l}\text { Voltage } \\
\text { Harmonics }\end{array}$ & \multicolumn{3}{|c|}{$\begin{array}{l}\text { As indicated in } \\
\text { EN 61000-2-2 }\end{array}$} & $10 \mathrm{~min}$ \\
\hline Unbalance & & $2 \%$ & $95 \%$ & \\
\hline Events & & 100 & $100 \%$ & \\
\hline
\end{tabular}

Within the parameter of events the dips and swells have been grouped together, because of their similarity. Although the norm clearly indicates limits for the parameters of frequency, voltage magnitude, flicker and voltage harmonics, for the remaining eight parameters it either only gives indicative values or makes brief comments.

Given that frequency is a parameter inherent to the electricity system, the distribution company cannot normally do anything about it, so it is not explicitly included in the measurement, although it will be monitored.

\section{Measurement procedure}

A measurement procedure establishes how, for how long, and where the optimum and most efficient measures are to be taken, bearing in mind the resources, size of the electricity network and variety of parameters to be measured.

\section{A. Duration of measurement periods}

Depending on the characteristics of the wave indicated by the norm EN 50160 and on their periods of measurement, two main groups of measurement plans are established: one weeklong and the other yearlong. Other special measurement plans are also considered, but which are not currently in force, including information signals, and voltage boosts.

Within the weeklong plans, the following data are included:

- Magnitude of the supply voltage (slow voltage variations).

- Voltage Unbalance.

- Harmonics.

- Interharmonics.

- Flicker.

The yearlong plans include:

- Voltage Dips.

- Overvoltages (Swells).

- Voltage Interruptions. 
Certain measurement equipment, such as electric wave quality analysers, can record the parameters of both time periods.

Of course if the equipment is rotated each week, then one cannot obtain an annual measurement. Because of the shortage of equipment, and to take advantage of these incomplete measurements, it was decided that annual measurements should be accumulated by zones.

\section{B. Equipment and measurement points}

The recently passed CEI norm 61000-4-30 establishes two classes of functioning according to the precision of the measurement and the specifications of the equipment.

Class A equipment is assigned to precise measurements at difficult points such as:

- Precise verification of compliance with norms.

- Settlement of disagreements between client and supplier.

- Especially problematic points of the networks.

Class B equipment corresponds to measurements which do not require so much precision, but simply a statistical indicator.

Given that class A equipment is more costly there is less available and it needs to be rotated between the measurement points mentioned above. At the same time, with the more numerous class B equipment, a survey is carried out, taking measurements at other points of the network chosen at random.

Once the initial class A measurements have been completed, then measurements are taken at those points where the survey detected worse voltage characteristics.

The distribution network in Burgos includes more than 3500 transformation centres (TCs) of Medium Voltage, with the division by zones and powers shown in Fig. 1.

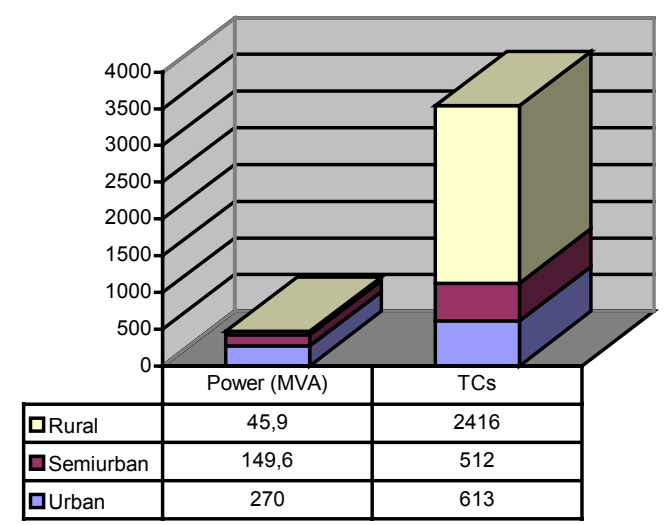

Fig. 1. TCs of Medium Voltage in the province of Burgos.

In our case we are focussing on an industrial zone near the capital, with nearly 90 TCs of medium voltage, since this is where we find the most important customers (greatest power contracted, and greatest continuity and product quality required).

During the elaboration of this work, only two class A electric wave analysers were available for the difficult points. This equipment is capable of recording all or almost all the parameters indicated in the norm EN50160. Given that the measurement periods are of a minimum of a week, we planned for an average of 6 measurements per month. At the time of writing this paper, we have more than 20 registers, most of which come from the semi-urban zone, and we hope to extend the study to $1 \%$ of each type of zone.

\section{Data collection}

Both in the weeklong plans and in the yearlong plans the equipment must be able to record the data with no loss of information. In the case of a week, the equipment is normally required to have sufficient storage capacity. At the end of the week the data are transferred to a computer or to a memory card.

In the case of yearlong measurements, there are two possible options. One is the remote and continuous transmission of data by the equipment via modem (not all such equipment is capable of doing this) or direct cable connection to a collection computer. The other option, which we chose to use, is to periodically download the data from the TC and empty the equipment's memory. This periodicity evidently depends on the equipment's capacity and implies having to accumulate the data from a single measurement point in the same file.

Once the measurements have been obtained at different points on the network, they are stored in a central computer. This central computer has the applications needed to create and administer the different databases and intermediate files.

\section{Data processing}

As shown in Fig. 2, the processing of data is composed of primary files, two applications, intermediate data files and four databases.

\section{A. Primary files}

The first problem to overcome is that the data recorded come from equipment made by different manufacturers, while even that from a single manufacture may have different storage formats. Normally, each piece of measurement equipment has specific software (DranViewC, Codam $\subset$, FlukeView $\subset$, etc.) which permits the exportation of data to an ASCII format file. Given that this format is recognised worldwide by any application and operative system, we will use this for the output and input of data in all the applications developed. 


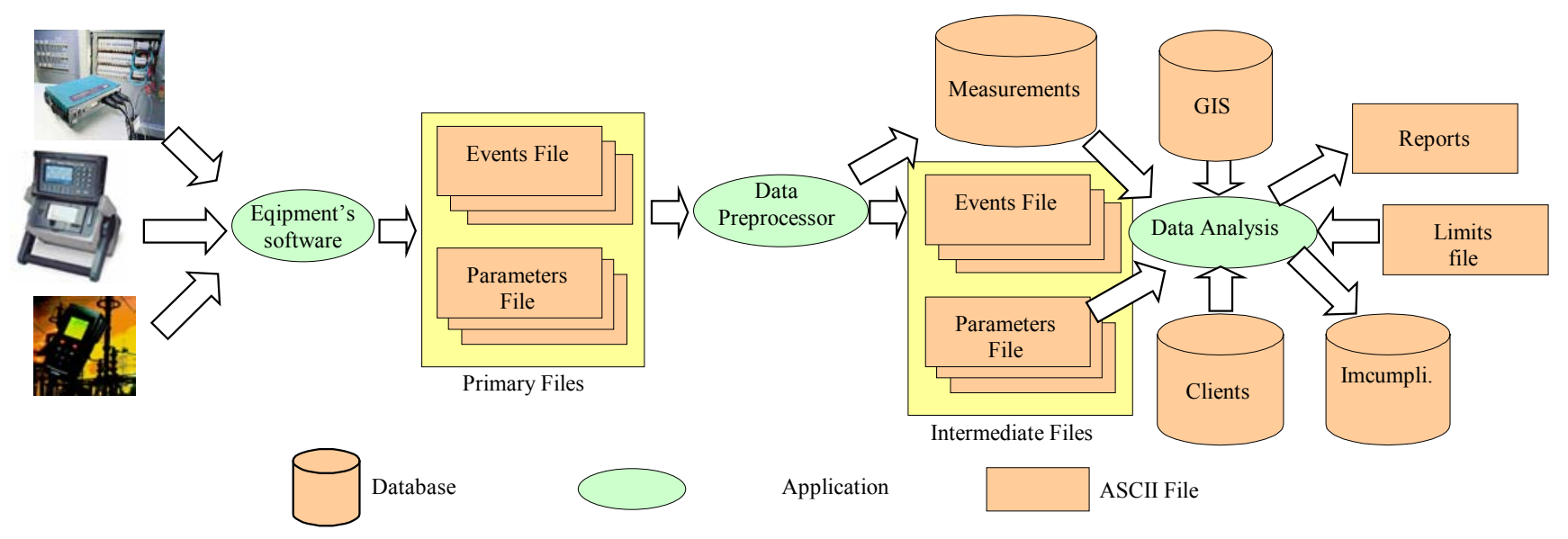

Fig. 2. Scheme of applications, files and databases.

The equipment's software creates the so-called primary files, which contain data relative to the different parameters which each piece of equipment can record. The names of both the primary files and the intermediate files are formed by the code assigned to the measurements taken, such that they can be directly related to each other. Normally, for an analyser of wave quality at least two files are needed: one of them we have called "parameters" and the other "events".

The parameter file, with extension .ppa, contains voltage data, powers, frequency, etc. These data are shown in columns with headings indicating the manufacturer's software, as can be seen in Fig. 3.

$\begin{array}{lrrrrr}\mathrm{X} & \mathrm{CHA} \text { Vrms(Volts) } & \mathrm{CHB} \text { Vrms(Volts) } & \mathrm{CHC} \text { Vrms(Volts) } & \mathrm{CHA} \mathrm{Hz}(\mathrm{Hz}) & \mathrm{CHA} \text { Vthd(\%) } \\ \text { 20/02/2003 12:38 } & 222,73 & 222,90 & 221,73 & 50,01 & 0,00 \\ \text { 20/02/2003 12:38 } & 222,81 & 222,83 & 221,77 & 50,01 & 0,00 \\ \text { 20102/2003 12:38 } & 222,73 & 222,90 & 221,73 & 50,01 & 1,56 \\ \text { 2/02/2003 12:39 } & 222,90 & 222,90 & 22,46 & 50,01 & 0,00 \\ \text { 20/02/2003 13:06 } & 219,56 & 220,86 & 220,56 & 49,98 & 0,00 \\ \text { 20/02/2003 13:06 } & 219,84 & 221,12 & 220,82 & 49,99 & 0,00 \\ \text { 20/02/2003 13:06 } & 219,84 & 221,12 & 220,82 & 49,98 & 1,14 \\ \text { 20/02/2003 13:07 } & 220,48 & 221,57 & 221,51 & 49,98 & 1,15 \\ \text { 20/02/2003 13:07 } & 220,66 & 222,02 & 222,05 & 49,99 & 1,05 \\ \text { 20/02/2003 13:08 } & 220,55 & 221,65 & 221,86 & 49,99 & 1,28\end{array}$

Fig. 3. Extract of Dranetz PP1 primary parameter file.

The events file, with extension .ppv, contains data relative to dips and swells, for which two magnitudes are required (size and duration).

\section{B. Data Preprocessor}

Once the primary files have been generated, a first application, denominated Data Preprocessor reads the files and from them generates two new intermediate files, one with data of measurements of parameters and the other of events, the two with a common format for all the measurements, independently of the equipment used to take the measurement. Given the variety of equipment and manufacturers, this application was developed using

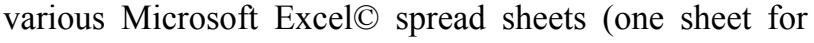
each equipment).

In addition, the application adds to a database of measurements a new record with the data of the new measurement, as we will explain in the following section.
Another operation performed by this application is to normalise the period of sampling of the equipment such that if it does not correspond with that indicated in the norm for a parameter, then the average of the values for the said period is calculated.

\section{Measurement database}

This Microsoft Access $\subseteq$ database contains two tables. The first table is that of measurements taken and contains the following fields:

- Code or identification of the measurement. Each measurement taken is coded with a name which includes the TC where it was taken

- Description.

- TC.

- Line.

- List of parameters recorded, according to the code used in the intermediate file.

- Start date/time and end date/time.

- Code of equipment used.

- Motive for the measurement. Complaint, random check or other motive.

- Person responsible.

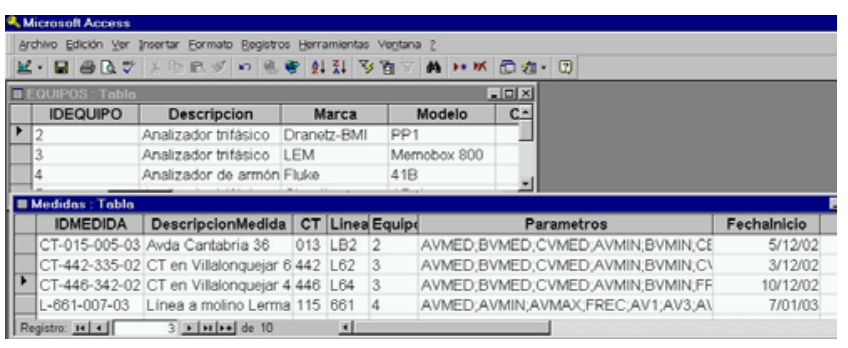

Fig. 4. Measurements database screen

The list of parameters measured is interesting, for example, if we have a flickermeter which only contributes measurements of efficient voltage values and of flicker or if we have equipment which only records events. 
The second table corresponds to the equipment available, with its make, model, class, calibration, characteristics, etc.

\section{Intermediate data files}

To create the intermediate files of parameters and of events (with extension .ipa and iev respectively) each magnitude susceptible of being measured was coded for later processing by the application of data exploitation known as Data Analysis.

For the parameter file, the magnitudes common to all the phases are composed of the first letter corresponding to the phase and/or neutral (A, B, C and D respectively). A second letter indicates whether it is a measure of voltage or current, power ( $\mathrm{V}, \mathrm{I}$ or $\mathrm{W}$ ) and then there is an indication of whether it is an average or maximum value, THD, a figure for the order of harmonics, etc. For magnitudes common to the three phases we have used terms such as FREC or DESEQ. Thus, for example, BVTHD refers to the rate of harmonic distortion of phase $\mathrm{B}$ and DV3 corresponds to the third voltage harmonics of the neutral (see Fig. 5).

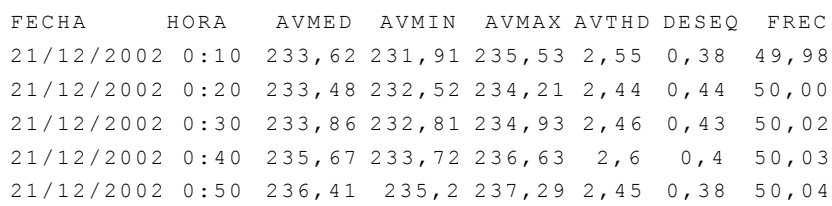

Fig. 5. Extract from an intermediate data file.

With respect to the events file we opted initially to group them in a single table, which includes (see Fig. 6) for each event, the phase and parameter in which it occurred, the maximum and minimum magnitude reached and its duration.

$$
\begin{array}{lcrrrrr}
\text { Fecha Hora } & \text { AV } & \text { TAV } & \text { BV } & \text { TBV } & \text { CV } & \text { TCV } \\
\text { 22/12/2002 } 13: 55 & & & 206,74 & 0,01 & & \\
\text { 22/12/2002 } 13: 55 & & & 205,74 & 0,01 & & \\
\text { 23/12/2002 10:21 } & & & & & 202,59 & 0,06 \\
\text { Total } & & 0 & 2 & & 1
\end{array}
$$

Fig. 6. Intermediate events file

The format used allows one to determine from the data exploitation application the duration according to that established in the norm for dips, where it is indicated that the duration of a dip commences when the first phase goes below the lower threshold and ends when the final phase exceeds it again (see Fig. 7, where one can see a duration of $720 \mathrm{~ms}$ ).

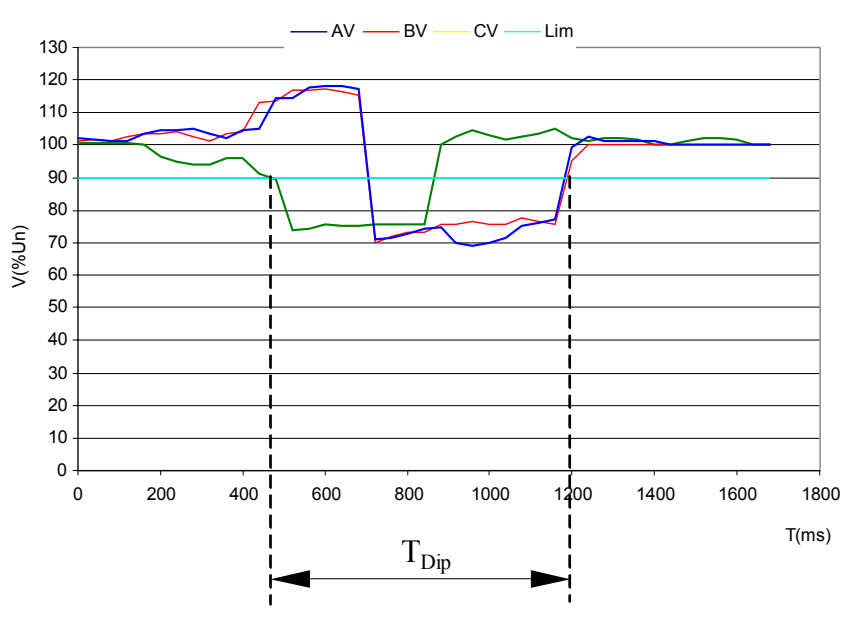

Fig. 7. Duration of the dips.

It is likely that more detailed information is required, in which the dips in each phase are analysed separately and particularities of some of the events are needed.

Depending on the recording capacity of the equipment used in the measurement one can obtain for a single dip various modes of storage: (i) using a table indicating the depth and duration or (ii) using all the series of data of the wave forms. As indicated in [6], the tabulation or recording of gaps in voltage is a point on which there is no international agreement and one of the most normal forms is to use the DISDIP table of UNIPEDE.

For example, the wave form shown in Fig. 8 can be recorded in two ways.

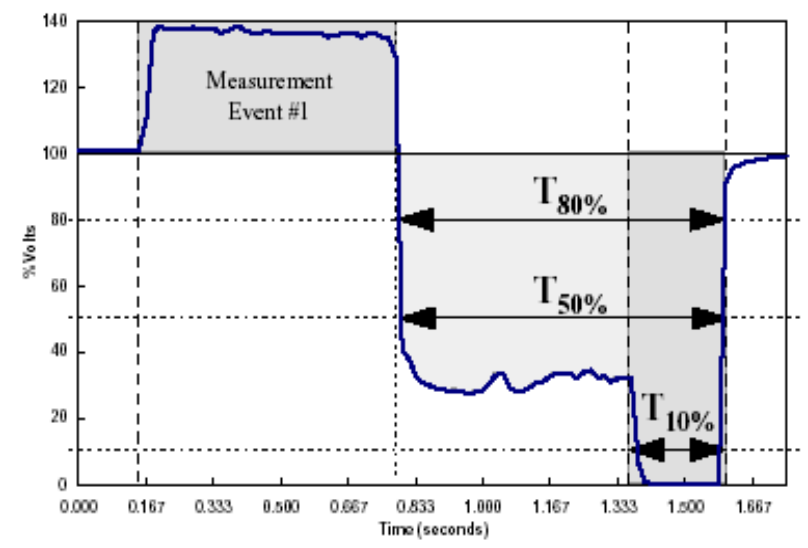

Fig. 8. Dip with steps.

The first would contain two events: a swell of approximately $40 \%$ of duration $650 \mathrm{~ms}$ and a dip of $100 \%$ of duration $850 \mathrm{~ms}$.

The second way would be that shown in table II, where one can appreciate that it provides more information than the previous method. 
TABLE II. DISDIP/UNIPEDE events.

\begin{tabular}{|l|l|l|l|c|l|l|l|l|}
\hline $\begin{array}{l}\text { Threshold } \\
(\%)\end{array}$ & $\begin{array}{l}<20 \\
\mathrm{~ms}\end{array}$ & $\begin{array}{l}20 . . \\
<100 \mathrm{~ms}\end{array}$ & $\begin{array}{l}100 . . \\
<500 \mathrm{~ms}\end{array}$ & $\begin{array}{l}0,5 . . \\
<1 \mathrm{~s}\end{array}$ & $\begin{array}{l}1 . . \\
<3 \mathrm{~s}\end{array}$ & $\begin{array}{l}3 . . \\
<20 \mathrm{~s}\end{array}$ & $\begin{array}{l}20 . . \\
60 \mathrm{~s}\end{array}$ & $\begin{array}{l}>= \\
1 \mathrm{~min}\end{array}$ \\
\hline$<=90$ & & & & 1 & & & & \\
\hline$<=85$ & & & & 1 & & & & \\
\hline$<=70$ & & & & 1 & & & & \\
\hline$<=40$ & & & & 1 & & & & \\
\hline$<=10$ & & & 1 & & & & & \\
\hline$>=110$ & & & & 1 & & & & \\
\hline
\end{tabular}

\section{E. Data Analysis}

The second application developed is the test bed to prove the usefulness of all the previous processes, formats and files and is responsible for generating all the information required by the distributing company. It can issue the official reports which will be required by the official organisms or obtain totally personalised reports for its own needs. Given that it must also handle other external databases such as the Geographical Information System (GIS) and the customer databases and must, in addition, have the capacity to make mathematical and statistical calculations, a special application has been developed in Visual Basic.

\section{Input data.}

As can be seen in Fig. 2, the main input data come from the data base of measurements taken from the intermediate data files and from a file of the different configurable limits between which the parameters must be found. In addition, the application can access and read the database of the company which contains information about TCs and customers and can relate them in this way to the measurements available.

\section{Reports and output data}

Using the data from the intermediate files, the application can perform a large variety of tasks for each individual measurement or for the whole, the main task being the elaboration of reports.

For each measurement, the data can be visualised graphically with their limits, extracting the values which surpass them. One can obtain graphs of accumulative frequency, etc. This is an important aid for the equipment's software.

The main criteria when generating a report may be: by zone, by disturbance, by period, by customer, or by equipment. When one wants to make a report, the applications access the database of the GIS to determine which TCs are included in the zone selected. With the information from the TCs the application accesses the database of measurements to select the measurements available for this zone and, for each measurement, the list of available parameters. Using a screen for configuration of reports, the data and graphs which must be included, together with their organisation, are specified (see Fig. $10)$.
In the graphical summary (see Fig. 9), a statistical analysis of all measured parameters is shown. From this analysis one can see which measured variables exceed the limit value as per limits file and/or what reserves are available.

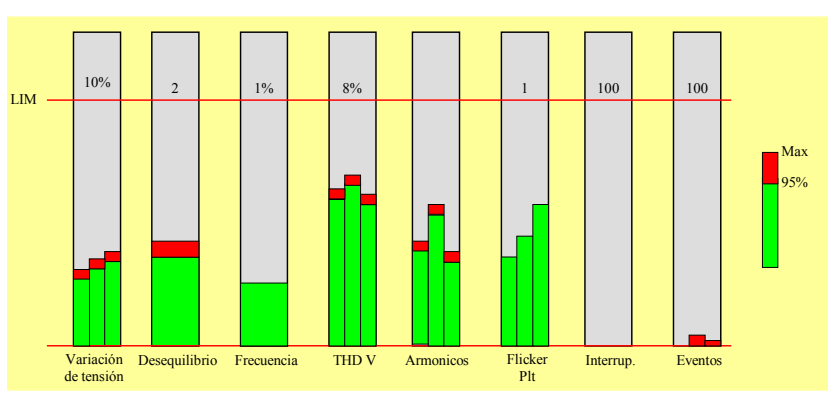

Fig. 9. Graphical summary.

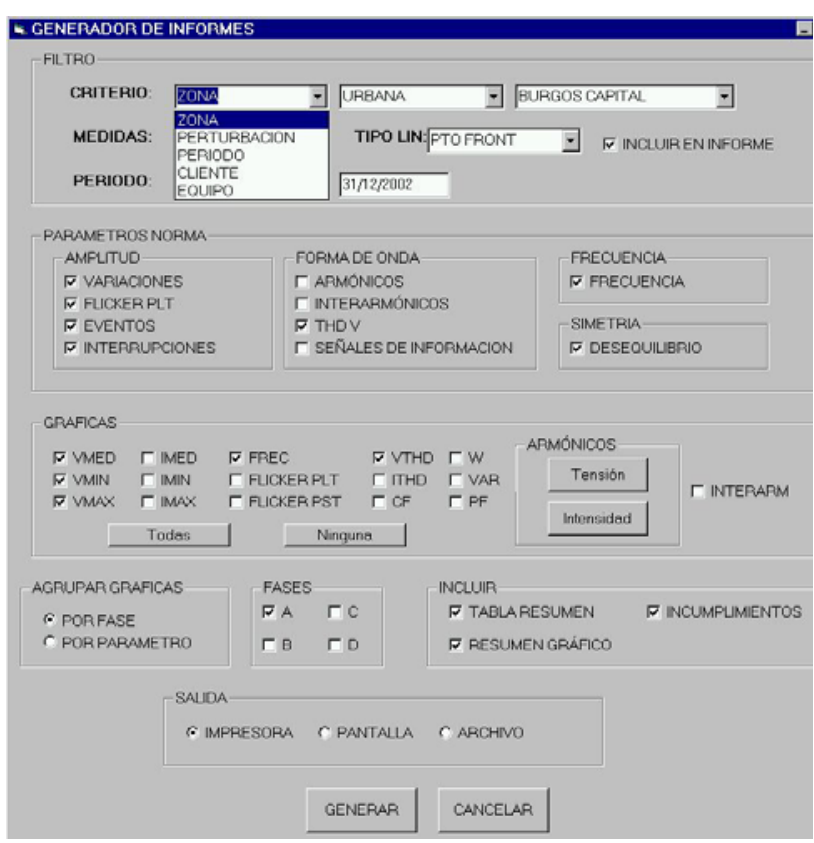

Fig. 10. Report configuration

\section{Data base of lack of compliance}

Given that the most interesting results are those of lack of compliance with the set limits, a special section has been added to deal with these and the application generates records in Microsoft Access $(C$ for the whole province. Likewise we are developing the option of being able to visualise in the graphic software of the GIS the TCs which fail to comply to determine possible relations between them and to be able to present them in the form of plans.

The database of lack of compliance allows one to establish the problematic points of the network and the calculations necessary to test compliance with the regulations. 


\section{Results and conclusions}

While awaiting the approval of procedures for product quality measurement, the present paper aims to determine various aspects necessary for these procedures. We have studied and developed the computer requirements and applications to process the data from different measuring equipment and to obtain a unified format for storage.

After several tests of binary formats (including PQDIF) we opted for the use of ASCII file data for parameters and events, which makes it possible to treat the data either from any spread sheet or from the application developed. This application can be used to test compliance with norms, generate reports, statistics, etc.

\section{Conclusion}

As regards wave quality in the province, although the number of measurements made is still low, it can be seen that the harmonics, voltage and flicker levels are almost always maintained below their maximum limits, as is indicated in the European study carried out by EURELECTRIC[8]. It can also be seen that the most frequent event is the gap in voltage lasting less than 20 ms and a depth of $15 \%$.

\section{Acknowledgements}

We wish to thank Ernesto Rozados, of the electricity company IBERDROLA S.A. for his collaboration and help in the elaboration of this paper.

\section{References}

[1] P1159 Working Group of the SCC-22 Power Quality Standards Committee. Draft: Recommended Practice for the transfer of Power Quality data. 2002.

[2] ROYAL DECREE 1955/2000, of 1 December, regulating the activities of transport, distribution, commercialisation, supply and procedures for authorisation of electrical energy installations.

[3] UNE-EN-50160. Characteristics of the voltage supplied by general distribution networks"

[4] UNESA. Guide to quality of electric Waves.

[5] IEC 61000-4-30. Testing and Measurement techniques. Power Quality Measurement Methods. February, 2003.

[6] D. Brooks, A.Sundaram, Voltage Quality Group of CIGRE. Recommendations for tabulating RMS variation disturbances with specific reference to utility power contracts. 1999.
[7] EN 61000-2-2. Compatibility levels for lowfrequency conducted disturbances and signalling in public low-voltage power supply systems.

[8] EURELECTRIC. Power Quality in European Electricity Networks. First Edition. 2002. 


\section{ANNEX \\ PART OF A REPORT FOR A MEASUREMENT}

\section{IBERDROLA BURGOS}

\begin{tabular}{|c|c|c|c|c|c|c|c|}
\hline \multicolumn{5}{|r|}{ Informe: } & \multicolumn{3}{|c|}{ Ref: $03-48-03$} \\
\hline \multicolumn{5}{|c|}{ Criterio: Medida } & \multicolumn{3}{|c|}{ Zona: Polígono de Villalonquéjar } \\
\hline \multicolumn{5}{|c|}{ Fecha Inicial: $21 / 12 / 02$} & \multicolumn{3}{|c|}{ Fecha Final: $28 / 12 / 02$} \\
\hline \multicolumn{8}{|c|}{ Medidas disponibles: } \\
\hline Num & Cod. & $\mathrm{CT}$ & Linea & Equipo & $\mathrm{U}_{\mathrm{n}}$ & Motivo & Responsable \\
\hline 1 & CT $-442-335-02$ & 0442 & B117 & MEMOBOX 800 & $230 \mathrm{~V}$ & Vidriera Burgalesa & Fernando Gárate \\
\hline
\end{tabular}

Gráficas por: FASE
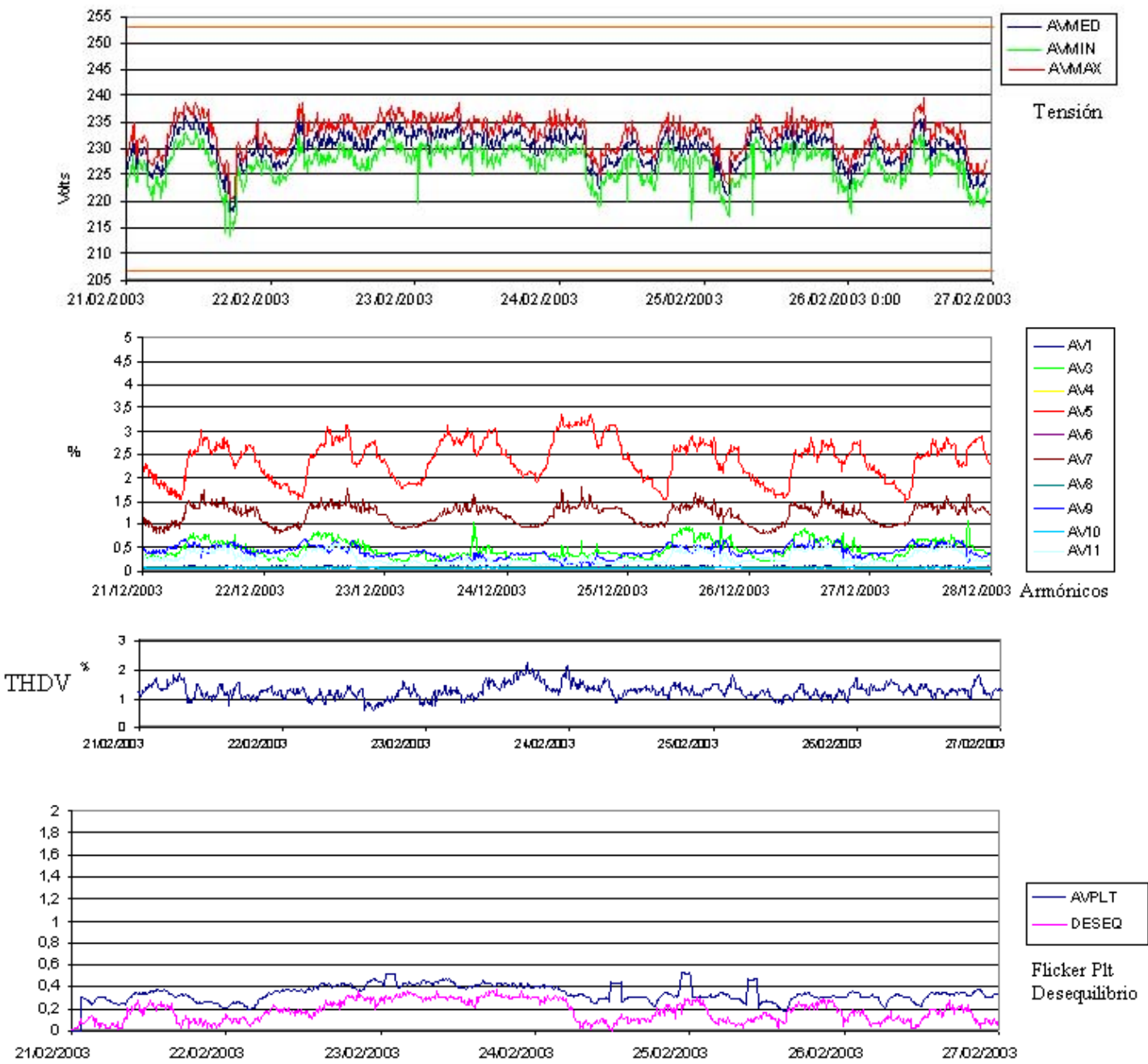

TABLA RESUMEN

\begin{tabular}{|l|c|c|c|c|c|r|r|r|}
\hline \multicolumn{4}{|c|}{ Parámetro } & \multicolumn{3}{|c|}{ Valor máximo } & \multicolumn{3}{c|}{ Valor 95\% } \\
\hline & Unidad & Limites & A & B & C & A & B & C \\
\hline Var. de Tensión & & $230 \mathrm{~V}$ & & & & & & \\
Max 100\%/95\% & $\%$ Un & $+10 \%$ & 3,07 & 3,29 & 3,84 & 2,46 & 2,53 & 3,15 \\
Min 100\%/95\% & $\%$ Un & $-10 \%$ & $-2,94$ & $-1,63$ & $-1,52$ & $-2,42$ & $-1,32$ & $-1,25$ \\
\hline Desequilibrio & $\%$ & 2 & \multicolumn{3}{|c|}{0,79} & \multicolumn{3}{|c|}{0,67} \\
\hline Flicker Plt & $\%$ & 1 & 0,54 & 0,51 & 0,47 & 0,52 & 0,48 & 0,46 \\
\hline THD V & $\%$ & 8 & & & & & & \\
En límites & $\%$ & 95 & 100 & 100 & 100 & 5 & 5 & 5 \\
Max. & $\%$ & & 3,48 & 3,71 & 3,66 & & & \\
\hline
\end{tabular}

